\title{
Two-channel Bilateral Control of Delta Robots with Gravity Compensation
}

\author{
Pablo René López Deras \\ Department of Electrical and Computer Engineering \\ Yokohama National University \\ Yokohama, Japan \\ lopez-rene-qa@ynu.jp
}

\author{
Yasutaka Fujimoto \\ Department of Electrical and Computer Engineering \\ Yokohama National University \\ Yokohama, Japan \\ fujimoto@ynu.ac.jp
}

\begin{abstract}
This paper presents three-dimensional bilateral delta robots based on a novel transfer-function-based two-channel bilateral control system. This configuration has proven to provide better control perfomance while only requiring two channels of communication, for position information exchange, as opposed to the traditional four-channel bilateral control method. The proposed three-dimensional bilateral Delta robot architecture proves to have good performance, however it is affected by gravity, and therefore, in order to ensure smooth operation of the delta robots we introduce a compensation for gravity on the bilateral control system, based on the analysis of the kinematics of the delta configuration. In this paper, we discuss the application of transfer-function-based two-channel bilateral control to three-dimensional delta robots, and the accuracy improvement obtained by the application of gravity compensation to the two-channel bilateral control.
\end{abstract}

Index Terms-bilateral control, delta robot, gravity compensation, haptics, motion control, two-channel

\section{INTRODUCTION}

$\mathbf{H}$ Aptics, to provide the sense of touching to the operator of a system beyond visual and auditory information, is needed for robots to support human activities fully and is expected to be widley applied to future robotic applications, for this reasons it has garnered attention of researchers recently [1]. Haptics has the potential of increasing productivity and precision of tasks while lowering the risk of forcing the system beyond its inteded uses. A control method that provides the means to realize haptics is Bilateral control, since it takes into consideradion a fast, real-time, information exchange flow.

Bilateral control systems are composed of a group of two or more subsystems, where one subsystem acts as the "master", receiving inputs from an operator, the master then exchanges position information with the other subsystems, called the "slaves", this other subsystems in turn replicate the position changes, and return the information of reaction forces from contact with the environment [2] [3].

Bilateral control that includes haptics transmision realization has been studied before and has been demonstrated to have highly accuarate haptics feedback from remote places [4] [5]. The most studied haptics transmision method is the bilateral control based on four-channel architecture. However, the four-channel architecture controllers must have position and force information designed independently, which increases the amount of data to transmit. This reasons have lead studies looking to reduce information, these studies propose a new method for designing a two-channel bilateral control system based on feedback of only position information without using reaction force estimation. The performance of two-channel bilateral control and four-channel bilateral control has been studied before [6], where it was confirmed that the twochannel bilateral control method has better performance while requiring less communication channels than the conventional four-channel in hard and soft environments with different characteristics as in free motion.

The two-channel control method parametrizes the controller by using a complementary sensitivity function, that can be designed in various ways, thus providing different performance results. This control method has been studied in one-degree of freedom environment [6]. The current paper proposes the extended study of the two-channel control method on a threedimensional environment, in order to achieve this the proposed environment is based on the delta robot architecture, and realizes the bilateral control by synchronizing the position and torque of motors in joint-space. Experiments executed in this proposed environment prove the novel method can be applied on a three-degree of freedom architecture, however, unlike the previous one-degree of freedom experiments, the proposed bilateral delta robots are affected by gravity, impairing the movement of the mechanism and accuracy of the method. Therefore a gravity compensation method [14] is also studied to improve on the accuracy of the proposed two-channel bilateral controled delta robots. This gravity compensation method is a non-mechanical solution based on the study of the Kinematics of the Delta robot architecture [8].

\section{Design of the Two-channel Bilateral Control}

Bilateral control seeks to synchronize the position between master and slave by having the slave system replicate the movements done in the master system, at the same time all reaction forces felt in the slave system due to interaction with the environment have to be felt in the master side, this is to say, there exists a law of action and reaction between the forces applied in both master and slave. This control objective can be expressed as the following equations. 


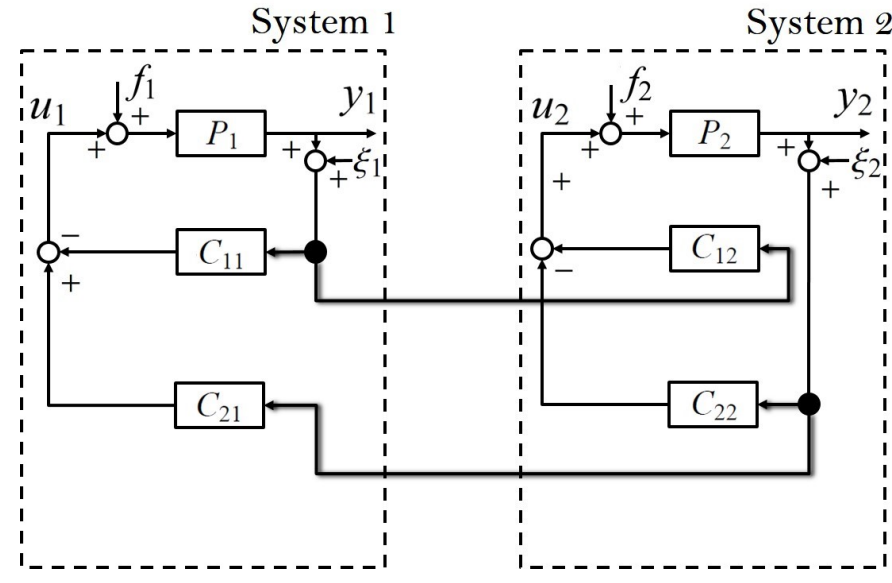

Fig. 1. Block diagram of two-channel bilateral control

$$
\begin{aligned}
& \text { Position: }\left\{\lim _{t \rightarrow \infty} y_{1}-y_{2}=0\right. \\
& \text { Force : }\left\{\begin{array}{l}
\lim _{t \rightarrow \infty} u_{1}-f_{2}=0 \\
\lim _{t \rightarrow \infty} u_{2}-f_{1}=0
\end{array}\right.
\end{aligned}
$$

where $y_{1}$ and $y_{2}$ represent the position of master and slave respectively. $u_{1}$ is the control output for the master side, while $u_{2}$ is the control output for the controller on the slave side. Finally $f_{1}$ and $f_{2}$ are the forces observed of master and slave respectively. Since the application of an external force $f_{1}$ in the controlled plant $P_{1}$ is answered with a force $u_{2}$ that has the same magnitude as $f_{1}$ applied to the controlled plant $P_{2}$, the realization of the conditions in (2) guarantees that the driving force in both controlled plants is the same.

With this objectives in mind a control system based on sensitivity function that only requires two channels of communication for exchanging position information can be derived. The sensitivity function $Q_{11}$ and $Q_{22}$ can be defined as the sensitivity of the transfer function from the external force $f_{1}$ to the output $y_{1}$ with respect to the parameter variation of the controlled plant $P_{1}$ and from $f_{2}$ to $y_{2}$ with respect to $P_{2}$. At the same time, we define two additional parameters $Q_{12}$ and $Q_{21}$, this two additional parameters represent the transfer functions from observation noise $\xi_{2}$ to position $y_{1}$ and from $\xi_{1}$ to position $y_{2}$ as follows

$$
\begin{aligned}
Q_{11} & =\left(A-1-C_{22} P_{2}\right) / A \\
Q_{22} & =\left(A-1-C_{11} P_{1}\right) / A \\
Q_{12} & =C_{21} P_{1} / A \\
Q_{21} & =C_{12} P_{2} / A \\
A & =\left(1+C_{11} P_{1}\right)\left(1+C_{22} P_{2}\right)-C_{12} C_{21} P_{1} P_{2} .
\end{aligned}
$$

The proposed system consists of four controllers, two for feedback control and two for information exchange, by solving (3)-(7) with respect to the controllers, we can derive the controllers parametrization as follows.
TABLE I

CONDITIONS THAT Q MUST SATISFY.

$$
\begin{aligned}
C_{11} & =\frac{1}{P_{1}} \frac{\left(1-Q_{22}\right) Q_{11}+Q_{12} Q_{21}}{\left(1-Q_{11}\right)\left(1-Q_{22}\right)-Q_{12} Q_{21}} \\
C_{12} & =\frac{1}{P_{2}} \frac{Q_{21}}{\left(1-Q_{11}\right)\left(1-Q_{22}\right)-Q_{12} Q_{21}} \\
C_{21} & =\frac{1}{P_{1}} \frac{Q_{12}}{\left(1-Q_{11}\right)\left(1-Q_{22}\right)-Q_{12} Q_{21}} \\
C_{22} & =\frac{1}{P_{2}} \frac{\left(1-Q_{11}\right) Q_{22}+Q_{12} Q_{21}}{\left(1-Q_{11}\right)\left(1-Q_{22}\right)-Q_{12} Q_{21}}
\end{aligned}
$$

Then by designing the complementary sensitivity function $Q$ the performance and complexity of the control system can be realized in a wide range of ways. To guarantee the internal stability of the system the $Q$ parameters should be designed through the conditions described in Table I. This way we can design $Q$ to simplify the control system, where only one controller is needed to realize feedback and information exchange. In our controller $Q$ was designed as follows to achieve this simplification.

$$
\begin{aligned}
Q_{11}= & Q_{22}=Q_{H}=\frac{s^{3}+a_{2} s^{2}}{s^{3}+a_{2} s^{2}+a_{1} s+a_{0}} \\
Q_{12}=Q_{21}=Q_{L} & =\frac{a_{1} s+a_{0}}{s^{3}+a_{2} s^{2}+a_{1} s+a_{0}} \\
Q_{H}+Q_{L} & =1
\end{aligned}
$$

Here $Q_{11}$ and $Q_{22}$ are designed as HPF with third-order Butterworth coefficients, and $Q_{12}$ and $Q_{21}$ are LPF. The transfer function for the two-channel method with one controller is then defined as follows.

$$
\begin{aligned}
& {\left[\begin{array}{c}
y_{1}-y_{2} \\
u_{1}+u_{2} \\
u_{1} \\
u_{2}
\end{array}\right]=} \\
& \left.0 \begin{array}{cccc}
0 & 1 & 1 \\
-Q_{H}+\frac{P_{1}}{P_{2}} Q_{L} & \frac{P_{2}}{P_{1}} Q_{L}-Q_{H}-\frac{Q_{H}}{P_{1}}+\frac{Q_{L}}{P_{2}} & \frac{Q_{L}}{P_{1}}-\frac{Q_{H}}{P_{2}} \\
-Q_{H} & \frac{P_{2}}{P_{1}} Q_{L} & -\frac{Q_{H}}{P_{1}} & \frac{Q_{L}}{P_{1}} \\
\frac{P_{1}}{P_{2}} Q_{L} & -Q_{H} & \frac{Q_{L}}{P_{2}} & -\frac{Q_{H}}{P_{2}}
\end{array}\right]\left[\begin{array}{l}
f_{1} \\
f_{2} \\
\xi_{1} \\
\xi_{2}
\end{array}\right]
\end{aligned}
$$

With this definition the position error converges to zero when not affected by observed noise and the output of the master's controller $u_{1}$ converges to the estimated force $f_{2}$, 


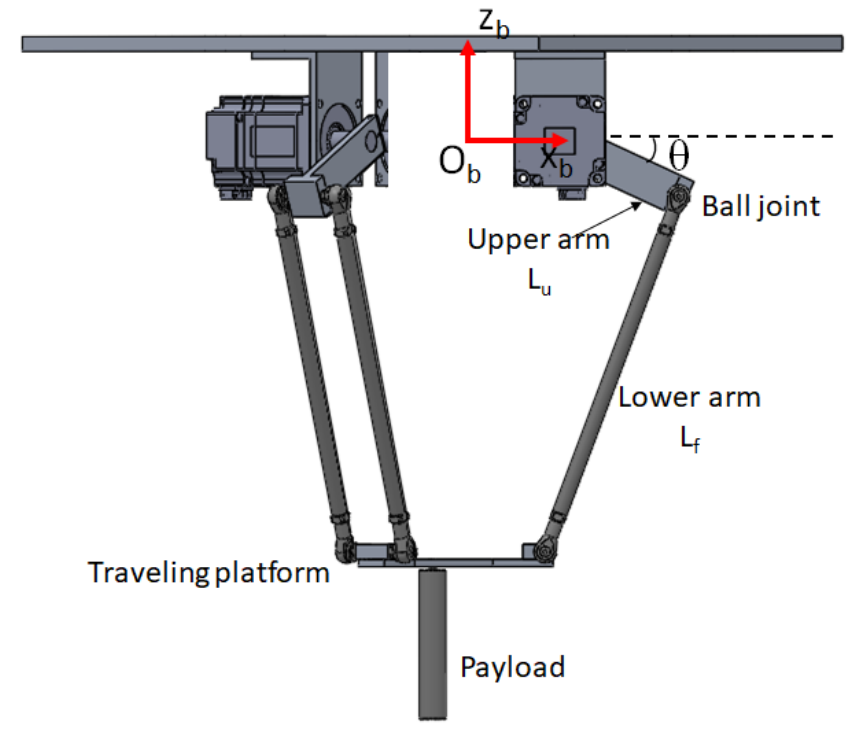

Fig. 2. Overview of delta robot parameters

similarly the output of slave's controller $u_{2}$ converges to the estimated force of the master $f_{1}$. This makes it possible to realize the objective of bilateral control and haptic transmission.

\section{GRAVITY COMPENSATION ON DELTA ROBOT}

The two-channel Bilateral Control method has been tested in a three-degree of freedom environment where it was confirmed that it realizes higher performance and requires less information exchange, thus allowing for faster exchange, than the four-channel bilateral control both in soft and hard environments. However the selected architecture, a Delta type parallel manipulator, is arranged in such a pattern that gravity affects its movement, and thus the operation of the experimental machine becomes harder on the side of the operator. In order to compensate gravity an approach based on the analysis of the Delta Robot Kinematics previously studied is proposed.

The Delta Robot architecture was selected since it has high accuracy, faster response, high stiffness and hight ratios of rigidity to weight, allowing for highly precise movements and transportation. The designed manipulator, shown in Fig. 3, has three revolute actuators mounted on a base having angle of separation as $120^{\circ}$ radians, each actuator has attached one of three parallel arms that converge in a traveling platform that will carry a payload serving as end effector. The parallel arms consist of two links, the upper link is attached directly to the actuators and is connected to the lower arms by ball joints, the lower arms consist of two parallel rods that form a parallelogram.

\section{A. General considerations}

The Forward Kinematic analysis of parallel manipulators are more complex that those of a the traditional serial manipulators [9]- [10]. The parameters needed for this analysis are presented in Fig. 2. In order to realize this analysis the following assumptions and considerations are made:
- Since the traveling platform always remains parallel to the base plate, the parallelogram arms can be regarded as a single rod arm without affecting the overall robot's kinematic behavior.

- The actuators, and ball joints are both placed in a circle, this assumption allows to treat the traveling platform as a single point $\mathrm{P}$ defined by the difference in the radius of the circle where the actuators lay and the circle where the traveling platform ball joints lay, defined then as:

$$
R=R_{b}-r_{p}
$$

- The reference frame is defined as $\left(O_{b}, X_{b}, Y_{b}, Z_{b}\right)$, the origin of this frame is the center of the circle where the revolute actuators are placed and it lays in the plane defined by the axis of revolution of the three actuators, positive $\mathrm{z}$ is pointing upwards and $\mathrm{x}$ is perpendicular to arm one.

- The angle of separation between the arms in the $x, y$ plane is $120^{\circ}$, therefore the angles for each arm are expressed as follows:

$$
\alpha=\left[\begin{array}{lll}
0 & \frac{2 \pi}{3} & \frac{4 \pi}{3}
\end{array}\right][\mathrm{rad}]
$$

\section{B. Forward Kinematics}

The forward kinematics, that is determining the $(x, y, z)$ position of the traveling platform in the frame of reference, when given the angle $\theta$ of the upper arm. This angle can be measured using encoders for each arm [11].

In order to find the position of the traveling plate the principle of trilateration can be applied [12], this principle is used to find a desired location using geometry of circles, spheres or triangles. For solving the FKP of the Delta robot consider there are three imaginary spheres with radius equal to the length of the lower arm $L_{u i}$ and centered in each of the ball joints between upper and lower arm for each arm $P b$. This spheres can be defined as follows:

$$
L_{u i}^{2}=\left(X_{t}-X_{0 i}\right)^{2}+\left(Y_{t}+Y_{0 i}\right)^{2}+\left(Z_{t}+Z_{0 i}\right)^{2}
$$

Where $X_{0 i}, Y_{0 i}$ and $Z_{0 i}$ represent the origin of each sphere, and are given by the following equations:

$$
\begin{aligned}
X_{0 i} & =\left(R+L_{u i} \cos \left(\theta_{i}\right)\right) \cos \left(\alpha_{i}\right) \\
Y_{0 i} & =\left(R+L_{u i} \cos \left(\theta_{i}\right)\right) \sin \left(\alpha_{i}\right) \\
Z_{0 i} & =L_{u i} \sin \left(\theta_{i}\right)
\end{aligned}
$$

Taking into consideration that the frame of reference has been selected to be in line with the first arm, the system of equations can be simplify as:

$$
\begin{aligned}
& L_{u 1}^{2}=\left(X_{t}-X_{01}\right)^{2}+Y_{t}^{2}+\left(Z_{t}-Z_{01}\right)^{2} \\
& L_{u 2}^{2}=\left(X_{t}-X_{02}\right)^{2}+\left(Y_{t}-Y_{02}\right)^{2}+\left(Z_{t}-Z_{02}\right)^{2} \\
& L_{u 3}^{2}=\left(X_{t}-X_{03}\right)^{2}+\left(Y_{t}-Y_{03}\right)^{2}+\left(Z_{t}-Z_{03}\right)^{2}
\end{aligned}
$$

This system can be solved by equating two spheres, this will result in a circle, and by intersecting this circle with the last 
sphere two possible intersection solutions are obtained, here the negative solution must be chosen, as the positive solution will be outside of the workspace of the Delta robot for the selected reference frame $O_{b}$.

\section{Inverse Kinematics}

The inverse kinematics refer to determining the angle $\theta_{i}$ for each actuator when given the position $\left(X_{t}, Y_{t}, Z_{t}\right)$ of the traveling platform. The inverse kinematics can have multiple solutions of $\theta_{i}$ for any given position $\left(X_{t}, Y_{t}, Z_{t}\right)$, thus it is necessary to define a criteria of decision for solving the IKP, the criteria used here is the angle $\theta_{i}$ that involves moving the manipulator as little as possible.

Then by analyzing the closure equations of the arms we can define the following equations for solving the IKP:

$$
\left\|P_{C_{i}} P_{b_{i}}\right\|^{2}-L_{f}^{2}=0
$$

Using this Inverse Kinematics the Jacobian matrix needed for compensating gravity can be derived [8], [13].

\section{Compensation method}

The gravity acting over the Delta robot can be separated into two components; the gravity affecting the traveling plate and the gravity affecting the actuated joints, each of this two types of gravities have to be compensated in order to fully guarantee gravity compensation. The gravity affecting the traveling plate is defined through the following equation.

$$
\tau_{G_{f} t p}=J_{c}^{T} m_{f t p}\left[\begin{array}{lll}
0 & 0 & -g
\end{array}\right]^{T}
$$

Where, $m_{f t p}$ is the summation of the masses affecting the bottom part of the robot: the mass of the payload $m_{p}$, the mass of the traveling platform $m_{t} p$ and the masses of the three lower arms, then $m_{f t p}=m_{p}+m_{t} p+\left(3 m_{f}\right)$. This gravity, however is represented in Cartesian space, thus the Jacobian matrix $J_{c}$ is needed to translate from Cartesian to Joint space.

The second component is the gravity affecting the upper part of the arms through its center of gravity, this component is defined with the following equation:

$$
\tau_{G_{u} f}=W_{u f} r_{G_{u} f}\left[\begin{array}{lll}
\cos \left(\theta_{1}\right) & \cos \left(\theta_{2}\right) & \cos \left(\theta_{3}\right)
\end{array}\right]
$$

Where $W_{u f}$ is the weight of the arms of the Delta robot, the mass the arms is calculated as the sum of masses from the upper arm, the ball-joints and two thirds of the fore arm masses times gravity, the equation of the weight is described as follows:

$$
W_{u f}=-m_{u f} g
$$

And $r_{G_{u} f}$ represents the center of gravity for the arm. The equation for this center of gravity is as follows:

$$
r_{G_{u} f}=L_{u}^{2} \frac{\frac{m_{u}}{2}+m_{b}+\frac{2 m_{f}}{3}}{m_{u}+m_{b}+\frac{2 m_{f}}{3}}
$$

Considering both 26 and 27 we can compensate the gravity affecting the Delta robot.

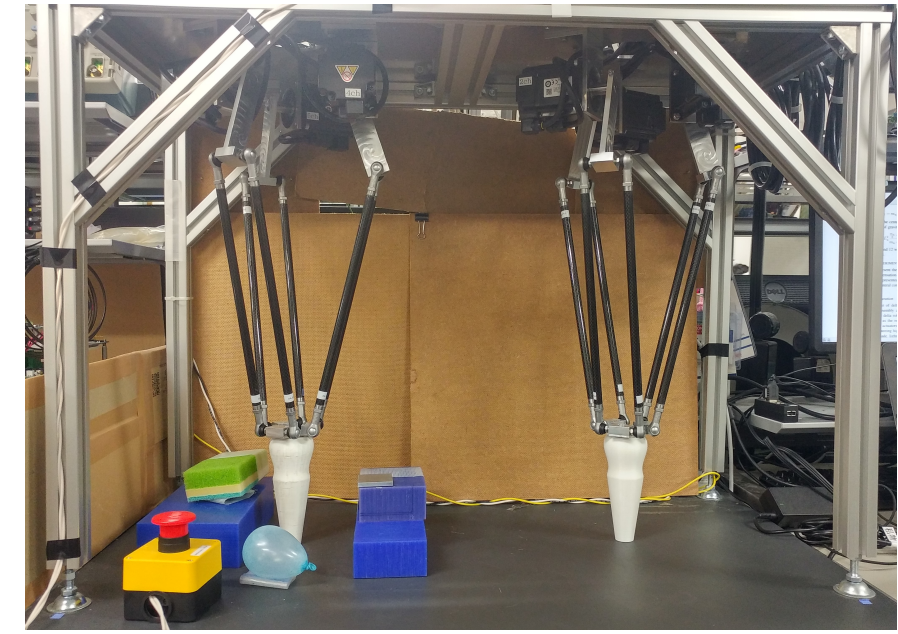

Fig. 3. Experimental configuration

TABLE II

PARAMETERS USED IN COMPENSATION METHOD.

\begin{tabular}{ccc}
\hline Parameters & Symbol & Value \\
\hline \hline Upper arm length & $L_{u}$ & $0.0920[\mathrm{~m}]$ \\
Lower arm length & $L_{f}$ & $0.3155[\mathrm{~m}]$ \\
Radius of base & $R_{b}$ & $0.0710[\mathrm{~m}]$ \\
Radius of traveling platform & $r_{p}$ & $0.0320[\mathrm{~m}]$ \\
Mass of ball joint & $m_{b}$ & $0.0143[\mathrm{~kg}]$ \\
Mass of upper arm & $m_{u}$ & $0.0585[\mathrm{~kg}]$ \\
Mass of lower arm & $m_{f}$ & $0.0300[\mathrm{~kg}]$ \\
Mass of traveling platform & $m_{t}$ & $0.0497[\mathrm{~kg}]$ \\
\hline
\end{tabular}

\section{EXPERIMENTAL RESULTS}

In this section we present the results of implementing the presented gravity compensation method in the two-channel bilateral control system presented in Fig. 3. The experiments are evaluated for the bilateral control system with and without gravity compensation.

\section{A. Experimental configuration}

The experimental pair of delta robots was designed with a policy of ease of assembly and reduction of weight for the moving parts. Each delta robot used consists of a set of three AC servo motors as the revolute actuators. The upperarms attached to this actuators are made from aluminum material A2017, guaranteeing high strength and high rigidity, the lower arms are made lighter by using a carbon pipe. The joints between the upper and lower arm and the lower arm and traveling platform are rod end bearings, this type of joint, as opposed to ball bearing, was chosen due to it being inexpensive and easy to manufacture. The traveling platform is made of the same aluminum material as the upper arms. Finally the end effector is a resin piece.

The parameters used in the experiments are shown in Table II. 


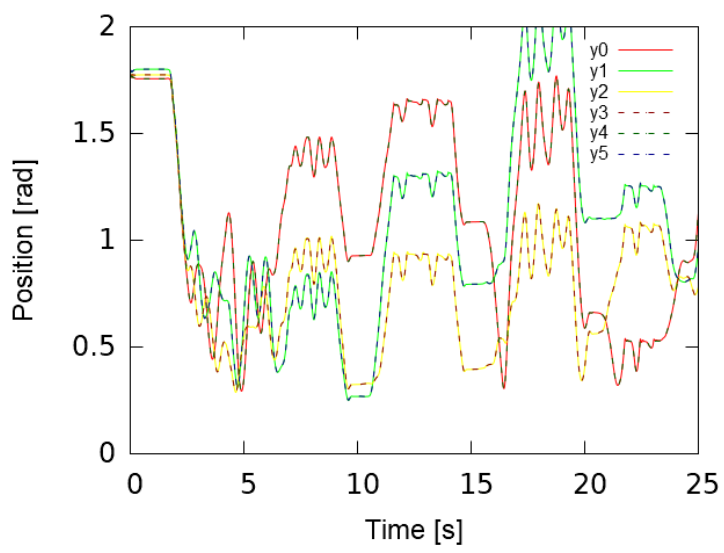

(a) Position results

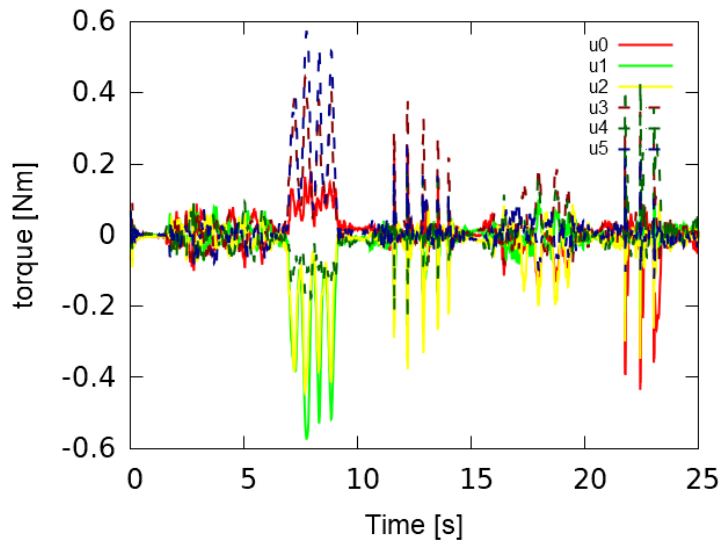

(b) Force results

Fig. 4. Experimental results of gravity compensation

\section{B. Discussion}

In this subsection we will describe the experimental results of applying the gravity compensation algorithm as opposed to the system without this gravity compensation.

Experimental results for the two-channel bilateral control with gravity compensation are show in in Figs 4(a)(b), where Fig 4(a) shows the results of position for each actuator in each master and slave, and Fig 4(b) shows the results of force for each actuator.

From this two plots it can be seen that the objective for bilateral control is achieved even when the gravity compensation algorithm is implemented, the slave follows the position of the master closely, even when engaged in gravity compensation on different environments. As for the force plot also shows that the input to the master system $u_{1}$ and the estimated reaction force from different environments is almost synchronized. This proves that even when the gravity is being compensated the operator can feel the environment that the slave is interacting with, regardless of the hardness of said environment, thus the control system still satisfies the law of action and reaction.

Figs $5(a)(b)(c)(d)$ show the position and force errors, the plots in Figs 5(a)(c) show the results for the system when gravity is being compensated, this results are further analyzed
TABLE III

RMS OF EACH MOTOR PAIR FOR GRAVITY COMPENSATED METHOD.

\begin{tabular}{ccc}
\hline System & RMS of position error & RMS of force error \\
\hline \hline Arm one & $1030.6 \times 10^{-6}[\mathrm{rad}]$ & $549.53 \times 10^{-6}[\mathrm{Nm}]$ \\
Arm two & $1374.316 \times 10^{-6}[\mathrm{rad}]$ & $537.788 \times 10^{-6}[\mathrm{Nm}]$ \\
Arm three & $1350.092 \times 10^{-6}[\mathrm{rad}]$ & $511.849 \times 10^{-6}[\mathrm{Nm}]$ \\
\hline
\end{tabular}

TABLE IV

RMS OF EACH MOTOR PAIR WITHOUT GRAVITY COMPENSATION METHOD.

\begin{tabular}{ccc}
\hline System & RMS of position error & RMS of force error \\
\hline \hline Arm one & $1473.943 \times 10^{-6}[\mathrm{rad}]$ & $546.116 \times 10^{-6}[\mathrm{Nm}]$ \\
Arm two & $1846.405 \times 10^{-6}[\mathrm{rad}]$ & $544.011 \times 10^{-6}[\mathrm{Nm}]$ \\
Arm three & $2022.733 \times 10^{-6}[\mathrm{rad}]$ & $545.33 \times 10^{-6}[\mathrm{Nm}]$ \\
\hline
\end{tabular}

in Table III where the root mean square of this errors is presented for each actuator pair and the plots in Figs 5(b)(d) show the error results for the system without gravity compensation, this are further analyzed in Table IV where the root mean square is presented for each actuator pair. In this figures it is shown that the gravity compensation algorithm does not add any noticeable error to the algorithm, thus the gravity algorithm can be used without decreasing the performance of the bilateral control method.

One of the proposed advantages of using a two-channel bilateral control is the reduced calculation time required for the control method. Table $\mathrm{V}$ shows the average sampling calculation time of the two-channel bilateral control with and without gravity compensation, from this results we can see that gravity compensation, since it does not require any information exchange does not increase calculation time in a significant manner.

\section{CONCLUSION}

This paper proposed bilateral delta robots based on jointspace two-channel bilateral control. The novel two-channel bilateral control method is applied in a three-dimensional environment by synchronizing the position and torque of the motors in joint-space. A gravity compensation method is also necessary to improve the accuracy of the method under the new architecture. Experiments on free movement and when in contact with different hard and soft environments composed of different materials where also done, proving a good accuracy when synchronizing the position and torque of master and slave robots.

\section{REFERENCES}

[1] K. Ohnishi, S. Katsura and T. Shimono, "Motion Control for Real-World Haptics," in IEEE Industrial Electronics Magazine, vol. 4, no. 2, pp. 1619, June 2010. doi: 10.1109/MIE.2010.936761

[2] C. D. Onal, "Bilateral control a sliding mode control approach," Master's thesis, Sabanci University, 2005. 


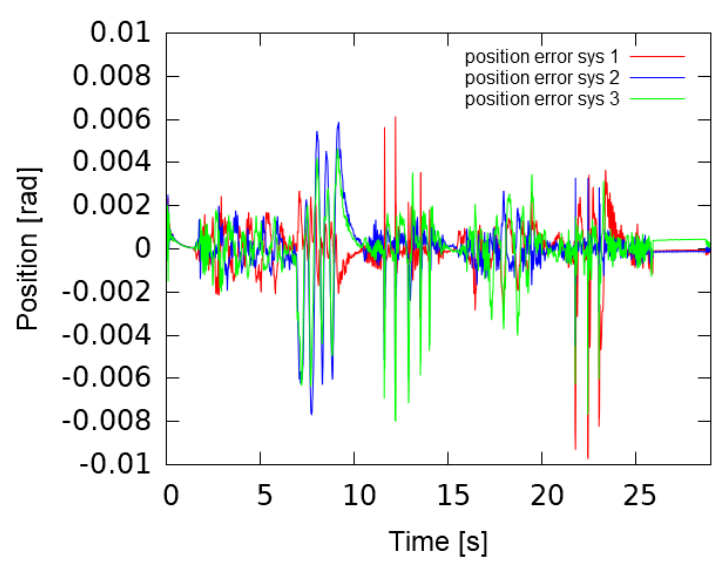

(a) Position error with gravity compensation

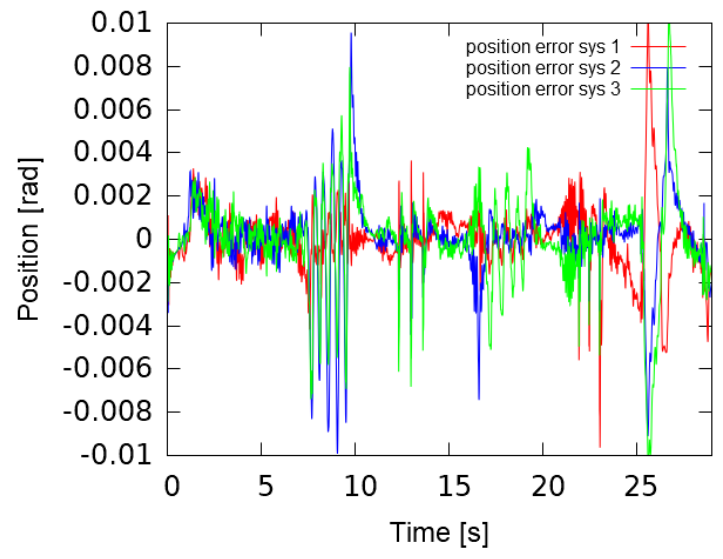

(a) Position error without gravity compensation

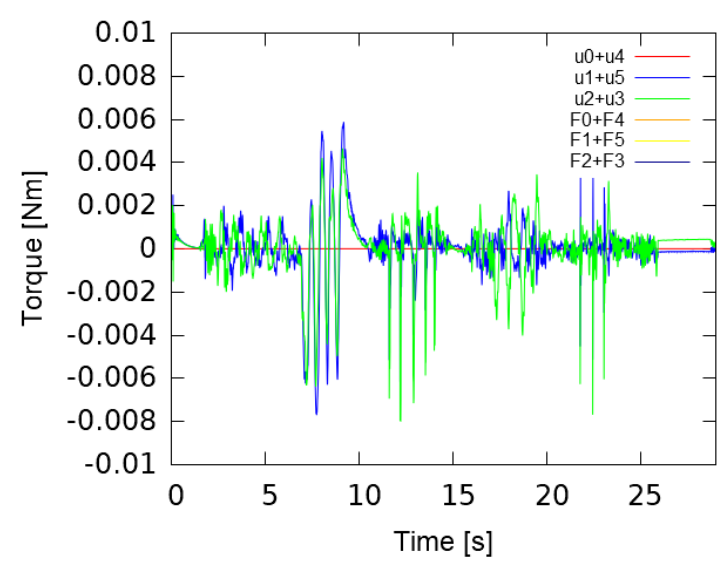

(b) Force error with gravity compensation

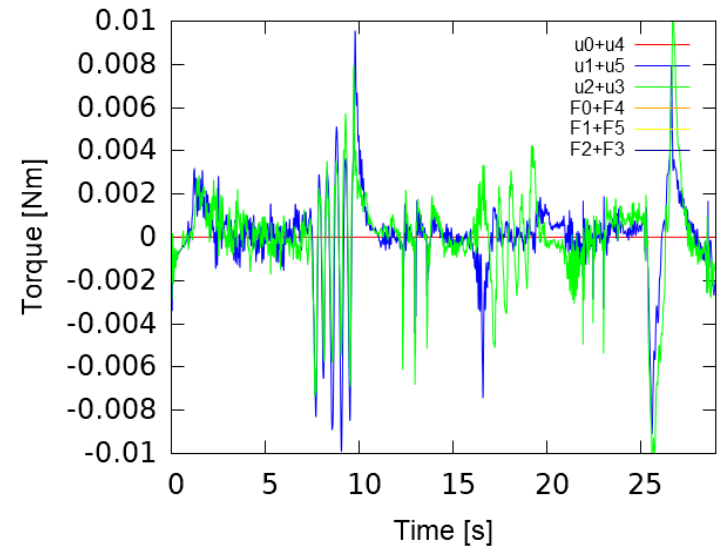

(b) Force error without gravity compensation

Fig. 5. Error comparison

TABLE V

CALCULATION TIME FOR CONTROL.

\begin{tabular}{cc}
\hline Condition & calculation time \\
\hline \hline Without gravity compensation [s] & $78.979 \times 10^{-6}$ \\
With gravity compensation [s] & $79.879 \times 10^{-6}$ \\
\hline
\end{tabular}

[3] T. Shimono, S. Katsura and K. Ohnishi, "Abstraction and Reproduction of Force Sensation From Real Environment by Bilateral Control," in IEEE Transactions on Industrial Electronics, vol. 54, no. 2, pp. 907918, April 2007. doi: 10.1109/TIE.2007.892744

[4] Y. Matsumoto, S. Katsura and K. Ohnishi, "An analysis and design of bilateral control based on disturbance observer," IEEE International Conference on Industrial Technology, 2003, 2003, pp. 802-807 Vol.2. doi: 10.1109/ICIT.2003.1290760

[5] S. Katsura, Y. Matsumoto and K. Ohnishi, "Realization of "Law of action and reaction" by multilateral control," in IEEE Transactions on Industrial Electronics, vol. 52, no. 5, pp. 1196-1205, Oct. 2005. doi: 10.1109/TIE.2005.855699

[6] M. Tajiri, P.López and Y. Fujimoto "Design of Two-channel Bilateral Control Systems by a Transfer-Function-Based Approach," in IEEE Transactions on Industrial Electronics, doi: 10.1109/TIE.2017.2750621, 2017.

[7] Masahiro Tajiri and Yasutaka Fujimoto, "Two-Channel Bilateral Control in Multi-Degree of Freedom Mechanism," proc. IEEJ International Workshop on Sensing, Actuation, Motion Control, and Optimization
(SAMCON), V-3, Nagaoka, 2017.03.

[8] C. Mitsantisuk and K. Ohishi, "Haptic human-robot collaboration system based on delta robot with gravity compensation," IECON 2016 - 42nd Annual Conference of the IEEE Industrial Electronics Society, Florence, 2016, pp. 5796-5801. doi: 10.1109/IECON.2016.7793735.

[9] V. Arakelian, "Gravity compensation in robotics," Advanced Robotics, vol. 30, no. 2, pp. 79-96, 2016. doi: 10.1080/01691864.2015.1090334

[10] A. D. Luca and S. Panzieri, "Learning gravity compensation in Robots: Rigid Arms, Elastic Joints, Flexible Links," International Journal of Adaptive Control and Signal Processing, Vol. 7, No. 5, Sep-Oct 1993, pp. 417-433.

[11] A. Olsson, "Modeling and control of a Delta-3 robot", M.S. thesis, Dept. Automat. Control, Lund University, Lund, Sweden, 2009.

[12] D. E. Manolakis, "Efficient solution and performance analysis of 3-D position estimation by trilateration," in IEEE Transactions on Aerospace and Electronic Systems, vol. 32, no. 4, pp. 1239-1248, Oct 1996. doi: 10.1109/7.543845.

[13] M. López, E. Castillo, G.García and A. Bashir, "Delta robot: inverse, direct, and intermediate Jacobians," Proceedings of the Institution of Mechanical Engineers, Part C: Journal of Mechanical Engineering Science, vol. 220, no. 1, pp. 103-109, 2006, doi: 10.1243/095440606X78263

[14] V. Arakelian, "Gravity compensation in robotics," Proceedings of Advanced Robotics, vol. 30, no. 2, pp. 79-96, 2016, doi 10.1080/01691864.2015.1090334. 